\title{
SOBRE LA POSIBILIDAD DE FORMULAR ESTÁNDARES DE PRUEBA OBJETIVOS*
}

\author{
Marina Gascón Abellán \\ Universidad de Castilla-La Mancha
}

\begin{abstract}
RESUMEN. La construcción de un estándar de prueba es una elección política o valorativa por la que se determina cuál de los siguientes dos errores en el conocimiento de los hechos se considera más asumible: a) aceptar como verdadero (o dar por probado) lo que es falso, o b) no aceptar como verdadero (o dar por no probado) lo que es verdadero. $\mathrm{La}$ manera de formular estándares de prueba objetivos dependerá del esquema de valoración (y por tanto del concepto de probabilidad) que se maneje; en los esquemas matemáticos se formularán cuantificando el grado de probabilidad exigible para probar un hecho, a su vez en los esquemas basados en el grado de confirmación será estableciendo exigencias o grados de confirmación. En suma, Los estándares de prueba se insertan en un proceso de valoración racional, y en consecuencia su papel de guías para la valoración (primero) y de esquemas para la justificación (después) será incompleto si esa valoración y justificación no se acompaña de los criterios racionales exigidos por la confirmación.
\end{abstract}

\begin{abstract}
The construction of a standard of proof is a political or evaluative choice that is used to determine which of the following two errors, when trying the facts, can be considered more acceptable: a) the admission of what is false as being true (considered proven), or b) the non admission of what is true as being true (or considered not proven). The way in which objective standards of proof are formulated will depend on the evaluation scheme (and therefore on the concept of probability) that has been chosen; in mathematical schemes the standards will be formulated by quantifying the degree of probability required in order to prove a fact, while in the schemes based on the degree of confirmation the standards will be formulated by establishing requirements or degrees of confirmation. In short, standards of proof fall within a rational evaluation process, and, as a result, the role they play as evaluation guides (firstly), and as schemes for justification (later), will be incomplete if both evaluation and justification are not carried out in accordance with the rational criteria required by the confirmation.
\end{abstract}

* El presente trabajo ha sido realizado en el marco del proyecto SEJ2005-08466/JURI, financiado por el Ministerio de Educación y Ciencia. 
Nota de la autora:

El presente trabajo tiene por objeto reflexionar sobre las posibilidades de formular estándares de prueba objetivos y sobre el alcance de tales fórmulas. Tiene su origen en la mesa redonda sobre "Valoración racional y estándares de prueba" que tuvo lugar en Tossa de Mar en el XI Congreso ítalo-español de teoría analítica del derecho, y en concreto en la lectura del texto principal presentado por Larry Laudan. No es, en sentido estricto, un comentario a este texto, pero ha estado altamente estimulado por él.

\section{Valoración racional, grado de probabilidad y estándares de prueba objetivos}

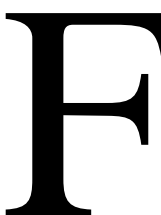

rente a ciertas concepciones de la prueba para las que el proceso no debe estar orientado a la verdad sino primaria y exclusivamente a la resolución del conflicto, doy aquí por supuesto que, por muchas razones, el proceso (o más en concreto la prueba) debe orientarse a averiguar la verdad. Sin embargo, el conocimiento de hechos que tiene lugar en la prueba judicial es imperfecto o relativo, lo que obedece básicamente a dos tipos de razones. De un lado, razones institucionales: la prueba judicial, en cuanto actividad encaminada a averiguar la verdad de los hechos relevantes para la causa, no es una actividad libre, sino que se desarrolla a través de un más o menos estricto sistema de reglas y cauces institucionales que muchas veces limitan y otras claramente impiden la consecución de ese objetivo. De otro lado, razones epistemológicas: el razonamiento probatorio está constituido básicamente por inferencias inductivas basadas en leyes probabilísticas o incluso por inferencias basadas en generalidades sin demasiado fundamento o sencillamente en prejuicios. Por las razones señaladas no puede suponerse que los resultados de la prueba garanticen la certeza absoluta, sino sólo un cierto "grado de certeza", o de probabilidad, sobre la verdad del enunciado probado. En definitiva, los resultados alcanzados en la prueba de un enunciado sólo pueden expresarse en términos de probabilidad ${ }^{1}$. Lo anterior, claro está, no significa que los resultados de la prueba no puedan tratarse como verdaderos; es más, hay buenas razones para esperar que el resultado de una prueba rigurosa sea fidedigno. Significa tan sólo que en sentido estricto lo más que puede decirse es que la prueba concluye con una hipótesis, un enunciado que aceptamos como verdadero aunque no sabemos si lo es o no, y que el grado de probabilidad suministrará un buen criterio para su justificación.

De hecho esta es la tarea a la que se enfrenta una valoración racional, es decir, una valoración que se desarrolla conforme al principio de libre

\footnotetext{
${ }^{1}$ He argumentado ampliamente sobre esta cuestión en Los Hechos en el Derecho. Bases Argumentales de la Prueba, Madrid, Marcil Pons, 2a ed. 2005.
} 
convicción, pero interpretando ésta no como convicción libérrima sino guiada por reglas racionales: si valorar consiste en evaluar si puede o no darse por probado un hecho controvertido, valorar libre y racionalmente consiste, más precisamente, en evaluar si el grado de probabilidad o de certeza alcanzado por la hipótesis que lo describe a la luz de las pruebas e informaciones disponibles es suficiente para aceptarla como verdadera. Por eso la principal tarea a la que se enfrenta una valoración racional es la de medir la probabilidad. Y por eso el objetivo de los modelos de valoración ha de ser proveer esquemas racionales para determinar el grado de probabilidad de las hipótesis, o su aceptabilidad.

Es en este contexto de valoración racional donde adquieren sentido y relevancia los estándares de prueba (en adelante SP), que son los criterios que indican cuándo se ha conseguido la prueba de un hecho; o sea, los criterios que indican cuándo está justificado aceptar como verdadera la hipótesis que lo describe. Teniendo en cuenta que esto ocurrirá cuando el grado de probabilidad o de certeza alcanzado por esa hipótesis se estime suficiente, la construcción de un SP implica dos cosas: a) en primer lugar implica decidir qué grado de probabilidad o certeza se requiere para aceptar una $\mathrm{H}$ como verdadera.; b) en segundo lugar implica formular objetivamente el SP; es decir, formular los criterios objetivos que indican cuándo se alcanza ese grado de probabilidad o certeza exigido. Y conviene enfatizar este último punto: un SP debe ser formulado conforme a criterios “objetivos”, pues -como afirma L.Laudan- un SP subjetivo, por ejemplo la intime conviction, no es en absoluto un $\mathrm{SP}^{2}$. Para que un SP tenga sentido debe poder expresarse mediante un criterio controlable.

Los SP se insertan pues en un proceso de valoración racional. Más exactamente, cumplen dos funciones. Una función heurística primero (en cuanto guías de una valoración racional): el SP es el criterio conforme al cual deberá el juez formular su valoración final sobre los hechos de la causa. Y una función justificadora después (en cuanto criterios para la motivación): el SP es el criterio conforme al cual ha de reconstruirse la justificación de la decisión probatoria.

\section{Niveles de exigencia: el SP como indicador del grado de probabilidad exigible}

La primera de las cuestiones implicadas en la construcción de un SP (¿qué grado de probabilidad o certeza se requiere para que pueda darse por probado un hecho?) no puede responderse de una manera concluyente, pues

${ }^{2}$ Vid. el trabajo de L.Laudan en este mismo volumen. 
no sólo depende de consideraciones epistemológicas sino también, y sobre todo, de cuestiones de policy.

Desde una perspectiva epistemológica cabe decir que la racionalidad exige un grado de probabilidad mínimo del que ningún orden jurisdiccional debería abdicar, a menos que estemos dispuestos a concebir la decisión probatoria como irracional. Ese grado mínimo lo constituye la probabilidad prevalente, que es el estándar de probabilidad normalmente exigido en el proceso civil. Según el estándar de la probabilidad prevalente (en adelante SPP) una hipótesis sobre un hecho resultará aceptable o probada cuando sea más probable que cualquiera de las hipótesis alternativas sobre el mismo hecho manejadas o consideradas en el proceso y siempre que dicha hipótesis resulte "más probable que no"; es decir, más probable que su correlativa hipótesis negativa. En consecuencia, en el caso de que sólo exista una hipótesis sobre el hecho en cuestión, el criterio de la probabilidad prevalente se resume en la regla "más probable que no"3. Con todo, cabe decir también que cuanto más exigente sea el estándar para probar un hecho, más racional será la decisión que declara probado ese hecho, pues un SP muy exigente minimiza la posibilidad de error y maximiza la posibilidad de acierto. Por eso, aunque el SPP es racional, resulta más racional un SP cualificado. Otra cosa es que pueda decirse -como hace Taruffo- que un proceso gobernado por un SP muy exigente no tiene como objetivo primario la búsqueda de la verdad, o más precisamente, la eliminación de errores, pues conforme disminuye la posibilidad de errar al declarar probado un hecho, aumenta la posibilidad de errar al darlo por no probado ${ }^{4}$.

Pero la construcción de un SP no depende sólo ni fundamentalmente de cuestiones de racionalidad, sino también y sobre todo de cuestiones de policy. Ello es así porque un SP establece una determinada distribución del error, y una distribución del error supone una determinada elección (político-valorativa) sobre la intensidad con que deben ser garantizados los derechos o intereses afectados por cada uno de los errores posibles.

En una decisión probatoria, en efecto, hay dos errores posibles:

- Error 1: aceptar como verdadero (o dar por probado) lo que es falso.

- Error 2: no aceptar como verdadero (o dar por no probado) lo que es verdadero.

No cabe duda que una decisión jurídica basada en una tesis fáctica errónea (sea por el Error 1 o por el Error 2) afecta derechos o intereses y/o

\footnotetext{
${ }^{3}$ Sobre las exigencias del estándar de la probabilidad prevalente, cfr. M.Taruffo, "Conocimiento científico y estándares de prueba judicial”, Jueces para la Democracia, 52, marzo de 2005, pp. 68-69.

${ }^{4} \mathrm{Cfr}$. El trabajo de Taruffo en este mismo volumen.
} 
defrauda expectativas legítimas. Por lo tanto, dependiendo de la importancia que se conceda a los derechos o intereses afectados por cada tipo de error, el SP será más o menos tolerante con ese tipo de error, y en consecuencia más o menos exigente. Son, pues, posibles tres situaciones.

A) Si se considera que los derechos o intereses afectados por los dos errores posibles merecen la misma protección (es decir, si se consideran igual de tolerables o asumibles ambos tipos de error), entonces el SP no será particularmente exigente y la probabilidad preponderante puede bastar.

B) Si se considera que los intereses afectados por el Error 1 merecen mayor protección que los afectados por el Error 2 (es decir, si se considera más tolerable o asumible el Error 2 que el Error 1), entonces el SP será particularmente exigente. La probabilidad prevalente no bastará y el SP exigirá una probabilidad cualificada. Esta es la razón por la que en el proceso penal, donde se considera que los derechos afectados por un eventual Error 1 (dar por verdadera o probada la hipótesis acusatoria) deben ser intensamente protegidos, se es muy poco tolerante con el Error 1 y en consecuencia se exige un SP muy cualificado.

C) Si, por el contrario, se considera que los derechos o intereses afectados por el Error 1 merecen menor protección que los afectados por el Error 2 (es decir, si se considera más tolerable o asumible el Error 1 que el Error 2), entonces el SP será poco exigente y podrá valer una probabilidad por debajo de la probabilidad preponderante.

En todo caso, la mayor o menor tolerancia con cada uno de los errores posibles es una cuestión de grado: se puede ser bastante tolerante con el error 1, o poco tolerante, o muy poco tolerante o nada tolerante. Y en consecuencia, puede haber una gradación de SP sobre un hecho, según los niveles de exigencia para dar por probado ese hecho (o de tolerancia con el Error 1).

En definitiva, un SP específico se construye decidiendo cuál de los dos errores posibles se considera preferible o más asumible (el de aceptar como verdadero lo que es falso o el de no aceptar como verdadero lo que es verdadero) y en qué grado estamos dispuestos a asumirlo. Y esta es, en última instancia, una elección política o valorativa.

\section{Formulación de los SP: esquemas de valoración}

Llegamos así a la segunda de las cuestiones implicadas en la construcción de un SP: la de formular el estándar; es decir, formular los criterios objetivos que nos indican cuándo puede darse por probado un (enunciado sobre un) hecho. Ahora bien, la formulación de un SP está muy vinculada a los esquemas de valoración que se manejen. Nos detendremos ahora en este punto previo. 
Existen al menos dos grandes esquemas o modelos de valoración racional de la prueba que suponen una distinta formulación de los SP: a) los basados en la aplicación de instrumentos matemáticos al proceso de valoración y b) los basados en esquemas de confirmación. Ambos modelos se corresponden con las dos grandes nociones de probabilidad: la matemática o estadística, que entiende que la probabilidad se predica de sucesos y se interpreta en términos de frecuencia relativa de la clase de eventos a la que pertenecen; y la lógica o inductiva, que se predica de proposiciones, y no de sucesos, y se corresponde con el uso común de "probablemente", "posiblemente", "presumiblemente" algo es verdad. Es decir, dicho concepto interpreta la probabilidad como una generalización de la verdad.

3.1. Quizá debido al gran desarrollo alcanzado por el cálculo matemático de probabilidades, no faltan los planteamientos que entienden que este cálculo es un buen instrumento para dar también cuenta de los procesos de razonamiento inductivo, aunque aplicando la probabilidad a enunciados en vez de a sucesos. El intento se ha realizado fundamentalmente con la aplicación del Teorema de Bayes a las inferencias jurídicas basadas en probabilidades subjetivas ${ }^{5}$. En su formulación más simple, el teorema afirma que la probabilidad de un evento $\mathrm{H}$ dado un evento $\mathrm{E}$, puede determinarse en función de la frecuencia estadística con la que dado $\mathrm{H}$ se verifica $\mathrm{E}$ y de la probabilidad atribuida precedentemente al evento $\mathrm{H}^{6}$. Así pues, la fórmula bayesiana permite medir el impacto que, sobre la probabilidad subjetiva previa del hecho que se pretende probar, provoca la introducción de ulteriores elementos de prueba. Su utilidad en el proceso reside -a juicio de los bayesianistas Finkelstein y Fairley- en que permite combinar información estadística sobre un cierto suceso con información no estadística7.

En el esquema matemático en general y en el bayesianismo en particular el SP se formula con absoluta precisión, indicando numéricamente el grado de probabilidad que debe alcanzar la hipótesis en cuestión (por ejemplo, 0.9), por debajo del cual no se considerará justificado aceptarla como verdadera. El problema es que este modelo presenta aún serias deficiencias y dificultades para fundar una teoría general de la valoración de la prueba, y

\footnotetext{
${ }^{5}$ La "probabilidad subjetiva” indica el grado de creencia personal de un factfinder racional sobre el acaecimiento de un suceso incierto.

$\mathrm{P}(\mathrm{E} / \mathrm{H})$

${ }^{6} \mathrm{P}(\mathrm{H} / \mathrm{E})=$------------- $\mathrm{P}(\mathrm{H})$

$\mathrm{P}(\mathrm{E} / \mathrm{No}-\mathrm{H})$

${ }^{7}$ M.O. Finkelstein y W.B. Fairley, “A Bayesian Approach to Identification Evidence”, Harvard Law Rev., 83, 1970. Sobre el bayesianismo pueden verse además los trabajos del simposio “Decision and Inference in Litigation”, Cardozo Law Rev., 13, 1991.
} 
por eso ha sido objeto de numerosas críticas. Algunas de estas críticas están animadas por consideraciones prácticas. Por ejemplo, llaman la atención sobre lo difícil que resulta para el juzgador cuantificar su valoración subjetiva previa; o sobre el riesgo que entraña poner en manos de los jueces instrumentos estadísticos incomprensibles para ellos. Además, la fórmula bayesiana supone un cálculo matemático relativamente simple en el casobase de tener que valorar una sola prueba que versa directamente sobre la hipótesis a probar, que es además una hipótesis simple. Pero la complicación del cálculo aumenta de manera impresionante cuando se usa para resolver situaciones más complejas, como la pluralidad de elementos de prueba relativos a una hipótesis, la cascaded inference (o prueba mediata) o la prueba de una hipótesis referente a un hecho complejo. Otras críticas, en cambio, ponen en cuestión la validez epistemológica del modelo en el campo judicial, en la medida en que conduciría a resultados contraintuitivos y resultaría dudosamente compatible con principios fundamentales del ordenamiento, como la presunción de inocencia ${ }^{8}$.

3.2. Pero racionalizar la valoración de la prueba no exige necesariamente la aplicación del cálculo matemático-estadístico. De hecho, la adopción de modelos matemáticos, quizás por las dificultades que presentan pero quizás también por la tradicional resistencia de los juristas a abrirse a conocimientos extrajurídicos, ha tenido escaso éxito, y los modelos de valoración racional de la prueba más aceptados (y que mayor predicamento han alcanzado) son los basados en esquemas de confirmación, que entienden que la probabilidad (lógica o inductiva) de una hipótesis depende del apoyo que le prestan las pruebas con las que está conectada a través de reglas causales. La probabilidad se mide aquí, no en términos de frecuencia relativa, sino de "grado de creencia”, “apoyo inductivo” o "grado de confirmación” de una hipótesis respecto de una información. Por eso, para facilitar la comunica-

\footnotetext{
${ }^{8}$ El blanco de las críticas lo constituye aquí el papel desempeñado por la probabilidad previa del hecho que se quiere probar. Si el valor atribuido a la misma fuera bajo, también sería bajo el resultado de la valoración final, con independencia de que la probabilidad indicada por el nuevo material probatorio fuese alta. Pero además, y conectado con ello, si la formulación de la probabilidad previa estuviera viciada de un error inicial, podría atentar contra principios básicos del ordenamiento, como la presunción de inocencia. En suma, el uso del teorema de Bayes produce una sobrevaloración de la probabilidad inicial y una minusvaloración del peso que tienen las nuevas pruebas en el cálculo de la probabilidad final. Para las deficiencias del bayesianismo vid. L.H.Tribe, “Trial by Mathematics”, Harvard Law Rev., 84, 1971; M.Taruffo, La prueba de los hechos, Madrid, Trotta, 2004; y H.L.Cohen, The Probable and the Provable, Oxford, Clarendon Press, 1977. Me remito también, en todo caso, a la atinada y detenida crítica de L. Laudan al bayesianismo, en su trabajo de este mismo volumen.
} 
ción y evitar confusiones, parece acertada la propuesta de R.D.Friedman ${ }^{9}$ de reservar el término "probabilidad" para la teoría convencional de la probabilidad matemática, y hablar en su lugar de grado de confirmación o de certeza. Por lo demás, el esquema valorativo del grado de confirmación es el que mejor se adecua a la estructura de los problemas probatorios con que el juez se encuentra: la existencia de una o varias hipótesis sobre los hechos de la causa y la necesidad de establecer, sobre la base de las pruebas disponibles, cuál de ellas resulta más aceptable o atendible.

Desde luego, en los esquemas de valoración basados en la probabilidad lógica o inductiva no contamos con la seguridad que proporcionan los números, por lo que los SP, en lugar de indicar numéricamente el grado de probabilidad que debe alcanzar una hipótesis sobre un hecho, deberán indicarnos cuándo está justificado aceptar dicha H como verdadera; es decir, cuáles son los criterios que establecen cuándo se ha conseguido la prueba de un hecho. Pues bien, puesto que la probabilidad lógica o inductiva de una $\mathrm{H}$ es equivalente a su grado de confirmación por las pruebas disponibles, cabe decir que una $\mathrm{H}$ podrá aceptarse como verdadera si no ha sido refutada por las pruebas y ha sido confirmada por éstas en grado suficiente. Por tanto, un SP se formulará mediante reglas o criterios de aceptabilidad de las hipótesis, que descansarán en última instancia en el grado de confirmación de las mismas. Y por eso un SP específico se caracterizará por exigencias de confirmación más o menos severas.

\section{Formulación de los SP: criterios objetivos para formular un SP}

\subsection{Dificultades de formulación de SP objetivos}

En el epígrafe anterior se ha dicho que la manera de formular SP objetivos dependerá del esquema de valoración (y por tanto del concepto de probabilidad) que se maneje: en los esquemas matemáticos los SP se formularán cuantificando el grado de probabilidad exigible para probar un hecho; en los esquemas basados en el grado de confirmación los SP se formularán estableciendo exigencias o grados de confirmación. Tiene sentido preguntarse ahora con qué fórmulas concretas se pueden expresar los SP. Pero antes, dos observaciones.

La primera es que, en realidad, el problema de encontrar fórmulas concretas para expresar un estándar no afecta a los SP basados en la probabilidad matemática, que se expresan cuantitativamente. El problema atañe sólo a los SP basados en la probabilidad lógica o inductiva; es decir, los propios de los esquemas valorativos del grado de confirmación, que sin embargo

9 “Assessing Evidence”, Michigan Law Rev., 94, 1996, p. 1821. 
-y como hemos dicho- parecen los más apropiados para dar cuenta de la prueba judicial.

La segunda es que, en la medida en que las fórmulas posibles para expresar un SP habrán de descansar en última instancia en exigencias o grados de confirmación, la búsqueda de estas fórmulas plantea dificultades. De una parte, porque, a diferencia de lo que ocurre con la probabilidad matemática, el grado de confirmación es difícilmente cuantificable, y por tanto la formulación del SP conforme a criterios objetivos se hace mucho más complicada. De otra, porque las situaciones probatorias pueden ser muy complejas y diferentes, y por tanto no valdría un criterio único para formular el SP: habría que establecer un criterio para cada una de ellas.

Para superar la primera dificultad es necesario no dejarse abatir por el “síndrome de los números”, por el empeño de que sólo vale lo que se puede acreditar con una precisión matemática. Por el contrario, hay que afrontar esta tarea con el objetivo (modesto, si se quiere, pero más factible) de encontrar fórmulas o criterios intersubjetivos para reconstruir la justificación de la decisión probatoria.

La segunda dificultad, por su parte, es una dificultad "empírica”: es realmente difícil construir formulaciones de un SP para cada una de las situaciones probatorias con las que el juez puede encontrarse. No obstante, $\mathrm{y}$ aunque las situaciones probatorias que pueden presentarse pueden ser muy variadas, a efectos de ilustrar cómo puede formularse un SP creo que podemos prescindir de este dato.

\section{2. ¿Con qué tipo de fórmulas se pueden expresar los SP?}

Por centrarme en el texto de Larry Laudan tomaré como referencia la prueba en el proceso penal. Laudan sugiere tres formulaciones de un SP para este proceso (A, B y C) como si fueran equivalentes o alternativas. En mi opinión esta visión no es correcta, pues, aunque pueda estarse de acuerdo con él en que las tres fórmulas (A, B y C) exigen que la hipótesis de la culpabilidad supere un Severe Test, cada una de ellas refleja un distinto grado de severidad o de exigencia, y por tanto un distinto SP, de manera que no son equivalentes sino que se optará por una u otra en función de lo exigente que se quiera ser para condenar. En otras palabras, aunque convengamos que en el proceso penal la hipótesis de la culpabilidad ha de superar un severe test, el severe test no es único. Hay distintos niveles de severidad o exigencia, y por tanto distintos SP, y la elección de uno u otro depende de lo exigente que se quiera ser para condenar; es decir, de la distribución de error por la que se haya optado, o si se quiere, de la intensidad con la que quieran protegerse los valores afectados por una eventual condena errónea. 
Formulación de estándares de prueba (según niveles de exigencia) para condenar.

Parto de la base de que cualquier SP para condenar exige que la hipótesis de la culpabilidad (en adelante Hc) no haya sido refutada. Pero, una vez cumplido este requisito, se podrían formular SP más o menos exigentes en virtud de los distintos grados de confirmación exigidos para la Hc y de los distintos grados de confirmación tolerados para la hipótesis de la inocencia (en adelante $\mathrm{Hi}$ ).

Para ilustrar esto podemos reconstruir los posibles grados de confirmación exigidos (para Hc) y tolerados (para Hi) asignándole el valor de 1 a la confirmación sólida, de 0.5 a la confirmación débil y de 0 a la ausencia de confirmación. Puede entenderse, a estos efectos, que hay una confirmación sólida cuando las pruebas, consideradas en su conjunto, sólo encuentran explicación si la hipótesis es verdadera $(\mathrm{p} \rightarrow \mathrm{h})$; o sea, cuando no son compatibles con la hipótesis contraria o sería muy difícil explicarlas si la hipótesis contraria fuera verdadera. Por el contrario, estaremos ante una confirmación débil cuando las pruebas puedan explicarse si entendemos que la hipótesis es verdadera, pero no son incompatibles con la hipótesis contraria $(h \rightarrow p)$.

Para simplificar las situaciones podemos representar los posibles grados de confirmación de Hc y de Hi del modo siguiente:

-Posibles grados de confirmación de Hc:

-Sólidamente confirmada: Hc-1

-Débilmente confirmada: Hc-0.5

-Posibles grados de confirmación de Hi:

-Sólidamente confirmada: Hi-1

-Débilmente confirmada: Hi-0.5

-Ausencia de confirmación: Hi-0

Pues bien, podemos reconstruir una escala de SP según los niveles de exigencia (o de severidad) para condenar. Los SP posibles, ordenados de menos exigente a más exigente, vienen caracterizados por el grado de confirmación exigido para Hc y por el grado de confirmación tolerado para Hi. Son los siguientes:

SP1: Hc-0.5 (exigido) y Hi-1 (tolerado)

SP2: Hc-0.5 (exigido) y Hi-0.5 (tolerado)

SP3: Hc-0.5 (exigido) y Hi-0 (tolerado)

SP4: Hc-1 (exigido) y Hi-1 (tolerado)

SP5: Hc-1 (exigido) y Hi-0.5 (tolerado)

SP6: Hc-1 (exigido) y Hi-0 (tolerado)

Creo que puede convenirse que el proceso penal exige una sólida confirmación de Hc. Por eso queda descartado el SP1, que es incluso irracional, 
el SP2, donde ni siquiera hay una clara prevalencia de la Hc, y el SP3, que supone que la Hc sólo está débilmente confirmada.

Sin embargo, los SP 4, 5 y 6 exigen todos una sólida confirmación para Hc, y por tanto en línea de principio podrían funcionar como estándares de prueba de Hc. La diferencia entre ellos está marcada por el distinto nivel de tolerancia a la confirmación de la Hi:

El SP4 es tolerante con la existencia de pruebas que confirmen (incluso sólidamente) la Hi. Por tanto, conforme a este SP se puede condenar aunque existan pruebas que sólo puedan explicarse si Hi fuera verdadera; o sea, pruebas que no son compatibles con Hc o que sería muy difícil explicar si Hc fuera verdadera. Me parece que este SP se correspondería con la regla C) de Laudan.

El SP5 exige que no existan pruebas que confirmen sólidamente la $\mathrm{Hi}$ pero es tolerante con la existencia de pruebas que confirmen débilmente Hi. Por lo tanto, según este SP se puede condenar aunque existan pruebas que sean compatibles con Hi (o que se puedan explicar si Hi fuera verdadera) pero que no son incompatibles con Hc. Me parece que este SP expresaría el mismo nivel de exigencia que la regla A) de Laudan.

Finalmente, el SP6 exige que no existan pruebas que confirmen ni sólida ni débilmente la Hi. Por tanto, conforme a este SP sólo se puede condenar cuando la Hi no goce de ningún grado de confirmación. Por el contrario, no se puede condenar si existen pruebas que apoyen o confirmen Hi aunque sea en grado mínimo; es decir, pruebas que puedan explicarse por (o sean compatibles con) la Hi.

\section{Sobre el alcance heurístico y justificatorio de los SP}

Es evidente que lo anterior no pretende ser, ni mucho menos, una propuesta elaborada de formulación de SP objetivos. Tan sólo tiene por objeto mostrar que los números no agotan la objetividad y que es posible ensayar SP objetivos en el ámbito de la probabilidad inductiva, es decir, recurriendo a los esquemas de confirmación.

De todos modos, aunque pudiéramos formular estándares de prueba con más o menos finura y precisión, conviene no exagerar ni el valor heurístico ni el valor justificatorio de un estándar. Respecto a lo primero, un SP es una guía para la valoración racional. Pero una guía limitada. En concreto, el valor de un SP -como señala reiterada y (creo) acertadamente Laudan- consiste en indicarle al juez lo que debe buscar en la prueba para poder después justificar su decisión, y por tanto la dirección en que debe buscar pruebas. Por ejemplo, si en el proceso penal el SP exige la sólida confirmación de la hipótesis acusatoria, lo que le indica al juez es que no debe escrutar (o interesarse por) la existencia de pruebas que simplemente confirmen o sean 
compatibles con dicha hipótesis, sino de pruebas que, de ser ciertas, no podrían explicarse si la hipótesis acusatoria fuera falsa. Pero no indica mucho más. El juez tendrá aún por delante la tarea de valorar esas pruebas y de atribuirles un determinado peso en la formación de su convicción, y el SP no ayuda en este punto. Respecto a lo segundo, un SP es el criterio conforme al cual ha de reconstruirse la justificación de la decisión probatoria. Pero tampoco es mucho más que eso. Ello significa que la justificación de la decisión será incompleta si no se justifica la concurrencia de los elementos que componen el estándar. Así, si el estándar fuera el SP5 y la decisión fuera condenatoria, no bastaría con decir que la Hc está sólidamente confirmada y la Hi no lo está: habría que justificar aún por qué la Hc está sólidamente confirmada (es decir, por qué las pruebas de cargo, consideradas en su conjunto, no podrían explicarse si Hi fuera verdadera) y la Hi, en cambio, no. Y para ello será necesario recurrir a lo que podríamos denominar "argumentos de la confirmación”; es decir, argumentos para sostener por qué está confirmada o no una hipótesis y con qué intensidad o grado está confirmada.

Estos argumentos de la confirmación, que son los argumentos para acreditar el grado o solidez de la confirmación (o si se quiere, la probabilidad inductiva de una hipótesis), derivan de la propia estructura de la confirmación, entendida como el apoyo que una prueba o una información presta a una hipótesis con la que está conectada a través de una regla causal. Así, en línea de principio, y sin ninguna intención de exhaustividad, podría decirse que el grado de confirmación de una hipótesis (o la solidez de la confirmación), aumenta o disminuye en función de los siguientes elementos: el fundamento cognoscitivo de las leyes causales que conectan las pruebas con la hipótesis (no es lo mismo que esas leyes causales gocen de un sólido fundamento científico, que sean genéricas e imprecisas máximas de experiencia, o que reproduzcan simples tópicos o prejuicios difundidos); la solidez epistemológica (o el grado de certeza) de las pruebas que la confirman (no es lo mismo que la hipótesis venga confirmada por el resultado de una prueba de $\mathrm{ADN}$ o por un testimonio no demasiado sólido); el número de pasos inferenciales que separan las pruebas de la hipótesis (no es lo mismo que la hipótesis venga confirmada por una prueba directa o por una prueba circunstancial); la cantidad de pruebas o confirmaciones (no es lo mismo que la hipótesis venga confirmada por una sola prueba, por algunas pruebas o por muchas pruebas); y la variedad de pruebas o confirmaciones, pues la variedad de pruebas proporciona una imagen más completa de los hechos (no es lo mismo que la hipótesis venga confirmada sólo por testimonios directos o que venga confirmada por testimonios directos, pruebas científicas y pruebas indiciarias). 
En definitiva, los estándares de prueba responden a la pregunta de cuándo se ha alcanzado la prueba de un hecho, o más precisamente, cuándo está justificado aceptar como verdadera la hipótesis que lo describe, y descansarán en última instancia en exigencias o grados de confirmación. Los argumentos de la confirmación, en cambio, son razones para justificar la solidez o la debilidad de la confirmación y responden, por tanto, a la pregunta de por qué una hipótesis está más o menos confirmada o resulta más o menos probable. Son también, retroactivamente, criterios a los que el juez debería atender para valorar el grado de confirmación que las pruebas atribuyen a una hipótesis. Los SP, en suma, se insertan en un proceso de valoración racional, y por eso su papel de guías para la valoración (primero) y de esquemas para la justificación (después) será incompleto si esa valoración y justificación no se acompaña de los criterios racionales exigidos por la confirmación. 
$\triangle \quad$ DOXA 28 (2005) 